\title{
A Reference Schema for the Unit Manufacturing Process Information Model
}

\author{
William Z. Bernstein ${ }^{1}$ and David Lechevalier ${ }^{2}$ \\ ${ }^{1}$ National Institute of Standards and Technology, Gaithersburg, MD 20899 \\ ${ }^{2}$ Engisis LLC, Bethesda, MD 20817 \\ william.bernstein@ nist.gov \\ david.lechevalier@engisis.com
}

Data DOI: https://doi.org/10.18434/M32027

Key words: ASTM E3012; process modeling; unit manufacturing process; XML schema.

Accepted: April 16, 2019

Published: May 7, 2019

https://doi.org/10.6028/jres.124.011

\section{Summary}

This document presents supporting documentation for a reference implementation of the Unit Manufacturing Process (UMP) information model presented in ASTM E3012, Standard Guide for Characterizing Environmental Aspects of Manufacturing Processes [1]. A version of this schema is used in the UMP Builder ${ }^{1}$ [2], a web-based toolkit for recording and storing UMP models.

\section{Data Specifications}

\begin{tabular}{|l|l|}
\hline NIST Operating Unit & Engineering Laboratory, Systems Integration Division, Life Cycle Engineering Group \\
\hline Format & eXtensible Markup Language (XML) Schema Definition (XSD) \\
\hline Instrument & Not applicable \\
\hline Spatial or Temporal Elements & Not applicable \\
\hline Data Dictionary & http://www.w3.org/2001/XMLSchema \\
\hline Accessibility & All datasets submitted to Journal of Research of NIST are publicly available. \\
\hline License & https://www.nist.gov/director/licensing \\
\hline
\end{tabular}

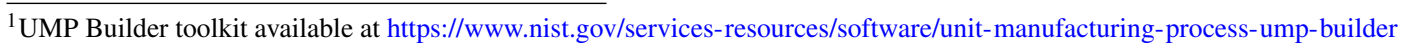




\section{Schema Documentation}

The root element of the schema is UnitManufacturingProcess . This element contains different attributes as follows:

- @name : (TYPE: xs:string, required): name of the represented UMP.

- QcreationDate : (TYPE: Xs:date, required): specifies the date of the model creation. The date is specified in the following form "YYYY-MM-DD" where YYYY indicates the year, MM indicates the month, DD indicates the day.

- Qversion : (TYPE: xs:int, optional): version number of the model.

- Oreviewed : (TYPE: xs:boolean, required): defines if the model has been reviewed. Value must be "true" or "false".

- Qtype : (TYPE: xs:string, required): specifies the type of process that is represented in this model. Refer to the taxonomy provided in the Manufacturing Processes Reference Guide by R. H. Todd, K. Allen and L. Alting [3]. If the process is not included in the taxonomy, suggest the lowest branch or node based on your expertise. Optional: Add your justification/reasoning in the description.

- Qdescription : (TYPE: xs:string, required): includes a general description of the UMP.

UnitManufacturingProcess contains different elements as follows:

- $[1 \ldots *]$ Author : includes the authors of the model.

- $[1 \ldots *]$ Keyword : includes the keywords for the model.

- $[1 \ldots *]$ Input : includes all inputs that enter the UMP such as material (for example, raw materials or work-in-progress), consumables (for example, lubrication or forced air), energy and external factors (such as temperature, humidity, particulates, vibration, and shocks) that occur during the manufacture of a product.

- $[1 \ldots *]$ Output : includes all outputs that exit the UMP model such as products, by-products, waste, and emissions.

- [1] ProductAndProcessInformation : includes relevant information to enable the transformation calculations of material, energy, and information. This includes items such as part geometry, material properties, control programs, and process plans.

- $[0 \ldots *]$ Resource : includes process resources such as equipment, fixtures, tooling, and inspection gauges.

- [1] Transformation : includes transformation equations (or descriptions), data driven models, and feasibility constraints that describe the relations between the inputs in the process.

- $[0 \ldots 1]$ ModelBound : includes formal or informal descriptions of the bounds of use for the model, providing guidance for the model's appropriateness or validity with respect to another situation.

- $[0 \ldots *]$ Review : includes the reviews of this model.

Author contains 4 elements: 
- [1] FirstName (TYPE: xs:string): the first name of the author.

- [1] LastName (TYPE: xs:string): the last name of the author.

- [1] Organization (TYPE: xs:string): the organization of the author.

- [0...1] Email (TYPE: xs:string): the email of the author.

Keyword is a xs:string type element. A Keyword must be unique.

Input and Output contain 5 elements and 2 attributes:

- [1] Name (TYPE: xs:string): the name of the input.

- [1] Symbol (TYPE: mml:math): the symbol represented in a MathML format [4].

- [1] Unit OR [1] UnitWithoutCode : the unit of measurement code identifying the input or output unit. Unit is used if a code is avalaible in the "Recommendation No. 20 - Units of Measure used in International Trade" from The United Nations Economic Commission for Europe (UNECE) [5]. UnitWithoutCode is used if no appropriate code is available in the "Recommendation No. 20 - Units of Measure used in International Trade" from UNECE.

- $[1 \ldots *]$ InputOutputBound : the equations to represent the bounds of the input or output.

- [0...1] Description (TYPE: xs:string): the description of the input or output.

- Qcategory (TYPE: xs:string, optional): a classification of the input or output.

- Qhyperlink (TYPE: xs:anyURI, optional): a hyperlink to provide supporting information about the input or output.

The Name of Input and Output must be unique.

ProductProcessInformation contains 5 elements:

- $[0 \ldots *]$ ControlParameters : includes tunable model parameters that can be adjusted to evaluate different process settings. Example: In machining processes, depthOfCut, spindleSpeed, and feedRate are traditionally classified as ControlParameters .

- $[0 \ldots *]$ FixedParameters : includes model parameters that are fixed through the evaluation of the transformation equations. Example: In machining processes, specificCuttingEnergy and density of the workpiece material are traditionally classified as FixedParameters .

- $[0 \ldots *]$ IntermediateVariable : includes calculated variables required to complete the evaluation of the metrics of interest. Example: In a milling process, the millingTime of a given cross sectional area of width and depth of cut must be calculated before assessing the machining power.

- $[0 \ldots *]$ Metric0fInterest : includes performance metrics that the model evaluates regarding the process. Example: Cost per part or $\mathrm{CO} 2$ emissions per part are classified as metrics of interest.

- $[0 \ldots 1]$ SupportingInformation : includes all other relevant links to information needed to instantiate the model, including production plans, product and engineering specifications, and setup-operation-teardown instructions, to name a few. 
ControlParameters and IntermediateVariable and MetricOfInterest contain 5 elements and 1 attribute:

- [1] Name (TYPE: xs:string): the name of the control parameter.

- [1] Symbol (TYPE: mml:math): the symbol represented in a MathML format [4].

- $[1 \ldots *]$ ParameterBound : the equations to represent the bounds of the control parameter.

- [1] Unit OR [1] UnitWithoutCode : the unit of measurement code identifying the control parameter unit. Unit is used if a code is avalaible in the "Recommendation No. 20 - Units of Measure used in International Trade" from The United Nations Economic Commission for Europe (UNECE). UnitWithoutCode is used if no appropriate code is available in the "Recommendation No. 20 - Units of Measure used in International Trade" from UNECE [5].

- [0...1] Description (TYPE: xs:string): the description of the control parameter.

- Chyperlink (TYPE: xs:anyURI, optional): a hyperlink to provide supporting information about the control parameter.

InputOutputBound and ParameterBound contain 2 elements:

- [1] MathMLEquation (TYPE: mml:math): the bound equation represented in a MathML format [4].

- $[0 \ldots 1]$ Description (TYPE: xs:string): the description of the bound equation.

Unit contains 1 element and 3 attributes that have fixed values:

- @listID (TYPE: xs:string, required): Value must be "UN/ECE Rec 20 Rev 3".

- QlistAgencyID (TYPE: xs:int, required): Value must be 6 .

- QlistVersionID (TYPE: xs:int, required): Value must be 3.

- [1] UnitCodeContent (TYPE: xs:normalizedString): the code representing the unit. Refer to UNECE code [5] to choose the appropriate code.

UnitWithoutCode contains 2 elements:

- [1] SuggestedUnitRepresentation (TYPE: xs:normalizedString): a suggested unit representation that is needed for the unit (example: $\frac{m o l}{\left(m^{3} \times s\right)}$ ).

- [1] SuggestedUnitCodeContent (TYPE: xs:normalizedString): a suggested code content that could be used for this unit. It can be a combination of existing codes from the "Recommendation No. 20 - Units of Measure used in International Trade" from UNECE [5].

FixedParameters contains 6 elements and 1 attribute

- [1] Name (TYPE: xs:string): the name of the fixed parameter.

- [1] Symbol (TYPE: mml:math): the symbol represented in a MathML format [4]. 
- [1] Value (TYPE: xs:decimal): the value to represent the bounds of the fixed parameter.

- $[0 \ldots *]$ ParameterBound : the equations to represent the bounds of the fixed parameter.

- [1] Unit OR [1] UnitWithoutCode : the unit of measurement code to define the fixed parameter unit.

- [0...1] Description (TYPE: xs:string): the description of the control parameter.

- @hyperlink (TYPE: xs:anyURI): a hyperlink to provide supporting information about the control parameter.

SupportingInformation contains 2 elements:

- $[0 \ldots *]$ Hyperlink

- [0...1] SupportingInformationDescription (TYPE: xs:string): external information (such as safety documentation, production plans, etc.) about the product and the process.

Hyperlink contains 1 element and 2 attributes:

- [1] Uri (TYPE: xs:anyURI): a hyperlink to provide supporting information about the product and process

- Qtitle (TYPE: xs:string, required): the title of the supportive information link

- Qdescription (TYPE: xs:string, optional): the description of the supportive information link Each Name of ControlParameters, FixedParameters, IntermediateVariable, and Metric0f Interest must be unique.

Resource contains 2 elements and 2 attributes:

- [1] Name (TYPE: xs:string): the name of the resource.

- [0..1] Description (TYPE: xs:string): the description of the resource.

- Qcategory (TYPE: xs:string, optional): a classification of the resource.

- @hyperlink (TYPE: xs:anyURI, optional): a hyperlink to provide supporting information about the resource.

The Name of Resource must be unique. Transformation contains 4 elements to represent the transformation occurring in the UMP:

- $[0 \ldots *]$ Equation

- $[0 \ldots *]$ PMMLModel

- $[0 \ldots *]$ FeasibilityConstraint

- [0...1] Description (TYPE: xs:string): the general description of the transformation. 
Equation contains 2 elements and 1 attribute:

- [1] MathMLEquation (TYPE: mml:math): the equation represented in a MathML format [4].

- [0..1] Description (TYPE: xs:string): the description of the equation.

- @name (TYPE: xs:string, required): name given to the equation for facilitate the identification of the equation.

- @hyperlink (TYPE: xs:anyURI, optional): a hyperlink to provide supporting information about the equation.

The value of @name must be unique.

PMMLModel contains 2 elements and 1 attribute:

- [1] DataDrivenModel (TYPE: pmml:PMML): the data driven model represented in a PMML format [6].

- [0..1] Description (TYPE: xs:string): the description of the equation.

- @name (TYPE: xs:string, required): name given to the equation for facilitate the identification of the PMML model.

- @hyperlink (TYPE: xs:anyURI, optional): a hyperlink to provide supporting information about the equation.

The value of @name must be unique. FeasibilityConstraint contains 2 elements and 1 attribute:

- [1] MathMLFeasibilityConstraint (TYPE: mml:math): the feasibility constraint represented in a MathML format [4].

- [0..1] Description (TYPE: xs:string): the description of the feasibility constraint.

- Oname (TYPE: xs:string, required): name given to the equation for facilitate the identification of the feasibility constraint.

- @hyperlink (TYPE: xs:anyURI, optional): a hyperlink to provide supporting information about the feasibility constraint.

The value of @name must be unique ModelBound contains 2 elements:

- [0...1] UseBoundDescription (TYPE: xs:string): the description to describe the use bounds of the model.

- $[0 \ldots *]$ UseBoundEquation

UseBoundEquation contains 2 elements:

- [1] MathMLEquation (TYPE: mml:math): the use bound equation represented in a MathML format [4]. 
- [0..1] Description (TYPE: xs:string): the description of the bound equation.

Review contains 2 elements and 1 attribute:

- [1] Reviewer

- [1] Feedback (TYPE: xs:string): the feedback of the model review.

- @creationDate (TYPE: xs:date, required): the date of the model review. The date is specified in the following form "YYYY-MM-DD" where YYYY indicates the year, MM indicates the month, DD indicates the day.

Reviewer has the same elements as Author:

- [1] FirstName (TYPE: xs:string): the first name of the reviewer.

- [1] LastName (TYPE: xs:string): the last name of the reviewer.

- [1] Organization (TYPE: xs:string): the organization of the reviewer.

- [0..1] Email (TYPE: xs:string): the email of the reviewer.

\section{Impact}

The purpose of the reference schema is to support the growth of the UMP repository, an effort to curate and exchange standardized representation of manufacturing process models.

\section{References}

[1] ASTM International (2016) ASTM E3012-16 - Standard Guide for Characterizing Environmental Aspects of Manufacturing Processes (ASTM International, West Conshohocken, PA). https://doi.org/10.1520/E3012-16

[2] Bernstein WZ, Lechevalier D, Libes D (2018) UMP Builder: capturing and exchanging manufacturing models for sustainability. ASME 2018 13th International Manufacturing Science and Engineering Conference (American Society of Mechanical Engineers), Vol. 1, p V001T05A022. https://doi.org/10.1115/MSEC2018-6331

[3] Todd RH, Allen DK, Alting L (1994) Manufacturing processes reference guide (Industrial Press Inc., New York).

[4] Ausbrooks R, Buswell S, David Carlisleand Stéphane Dalmas SD, Angel Diaz MF, Ion RHP, Kohlhase M, Miner R, Nico Poppelier BS, Soiffer N, Sutor R, Watt S (2003) Mathematical Markup Language (MathML) Version 2.0 (World Wide Web Consortium, Boston, MA), W3C Recommendation 21 October 2003, Second Edition. Available at https://www.w3.org/TR/MathML2/.

[5] United Nations Economic Commission for Europe (2005) TRADE/CEFACT/2005/24 - Recommendation No. 20, Unit of Measure used in International Trade (UNECE, Geneva, Switzerland).

[6] Data Mining Group (2016) Predictive Model Markup Language (PMML). Available at http://dmg.org/pmml/v4-3/GeneralStructure.html

About the authors: William Bernstein and David Lechevalier produced this schema in accordance with the ASTM E3012 conceptual model. The National Institute of Standards and Technology is an agency of the U.S. Department of Commerce. 